\title{
Analgesia before Performing Subarachnoid Block in the Sitting Position in Patients with Proximal Femoral Fracture: A Comparison between Fascia Iliaca Block and Femoral Nerve Block
}

\author{
Ghimire A, Bhattarai B, Koirala S, Subedi A
}

Department of Anaesthesiology and Critical Care

B.P. Koirala Institute of Health Sciences

Dharan, Nepal.

\section{Corresponding Author}

Ashish Ghimire

Department of Anaesthesiology and Critical Care

B.P. Koirala Institute of Health Sciences

Dharan, Nepal.

E-mail: ashishghimi@hotmail.com

\section{Citation}

Ghimire A, Bhattarai B, Koirala S, Subedi A. Analgesia before Performing Subarachnoid Block in the Sitting Position in Patients with Proximal Femoral Fracture: A Comparison between Fascia lliaca Block and Femoral Nerve Block. Kathmandu Univ Med 2015;50(2):152-5.

\section{ABSTRACT \\ Background}

Positioning for subarachnoid block (SAB) in patients with femoral fracture is painful and may remain suboptimal requiring use of large doses of opioids. These patients generally being elderly with multiple comorbidities and frailty are likely to have many undesirable effects of opioids including respiratory depression and confusion.

\section{Objective}

The objective was to compare the feasibility and effectiveness of fascia iliaca compartment block (FICB) and femoral nerve block (FNB) in reducing pain associated with positioning for subarachnoid block in patients undergoing proximal femoral fracture fixation procedures.

\section{Method}

Group FICB patients ( $n=15$ ) received fascia iliaca block with $30 \mathrm{ml}$ of $1.5 \%$ lignocaine with adrenaline and group FNB patients $(n=15)$ received femoral nerve block with 15 $\mathrm{ml}$ of $1.5 \%$ lignocaine with adrenaline. After the study blocks, patients were kept on supine position for at least 20 minutes before shifting them to the operation theatre. Pain was assessed by using visual analog scale values before the block and during the position for subarachnoid block. Time to perform subarachnoid block, quality of positioning and acceptance was recorded.

Result

Visual analog scale values during positioning for $S A B$ were lower in FIB group than in FNB $(1.0 \pm 1.1$ versus $2.1 \pm 0.8 ; P<0.05)$. Time to perform $S A B$ was shorter in FIB than in FNB $(109.6 \pm 28.2$ seconds versus $134.8 \pm 31.9$ seconds; $P<0.05)$. Quality of patient positioning for $S A B$ was comparable between the groups. Patient acceptance was less in group FNB $(\mathrm{P}<0.05)$.

\section{Conclusion}

Fascia iliaca compartment block provides better analgesia than femoral nerve block in terms of facilitating optimal positioning for subarachnoid block in patients undergoing proximal femoral fracture fixation procedure.

\section{KEY WORDS}

Fascia iliaca compartment block, femoral nerve block, positioning pain, subarachnoid block 


\section{INTRODUCTION}

Subarachnoid block (SAB) is commonly used and preferred technique of anaesthesia for fixation of proximal femoral fracture. However, positioning for $S A B$ in patients with femoral fracture is painful and may remain suboptimal requiring use of large doses of opioids. These patients generally being elderly with multiple comorbidities and frailty are likely to have many undesirable effects of opioids including respiratory depression and confusion. ${ }^{1,2}$

Local anaesthetic techniques including fascia iliaca compartment block (FICB) and femoral nerve block (FNB) have been shown to reduce pain associated with proximal femoral fractures. However, their effectiveness during positioning for $S A B$ have not been studied well and yet remain to be routinized. Both the techniques can be performed in any setting blindly using landmark approach. $^{3-5}$

The present study aimed to compare the feasibility and effectiveness of FICB and FNB in reducing pain associated with positioning for $S A B$ in patients undergoing proximal femoral fracture fixation procedures.

\section{METHODS}

The study was carried out from September 2011 to March 2012. After obtaining institutional approval and written informed consent, 30 ASA physical status I and II patients suffering from an isolated proximal femoral fracture who were to undergo open reduction and internal fixation were prospectively included in the study. Patients with hemorrhagic diathesis, peripheral neuropathies, allergy to amide local anaesthetics, mental disorders, and those on analgesics within 8 hour prior to performing $S A B$ were excluded from the study. All the patients were pre medicated with oral diazepam in the dose of $0.2 \mathrm{mg} /$ $\mathrm{kg}$ (not exceeding $15 \mathrm{mg}$ ) the night before and 2 hours prior to surgery. On arrival in the operation theatre on patient trolley while the skeletal traction was maintained, an 18 or 16 gauge intravenous cannula was secured into a large vein on the dorsum of the hand under local anaesthesia. All patients were preloaded with $500 \mathrm{ml}$ of ringer's lactate. Patients' heart rate $(H R)$, non invasive arterial blood pressure (NIBP), respiratory rate (RR), arterial blood saturation by pulse oxymetry $\left(\mathrm{SPO}_{2}\right)$ were noted after attaching the monitor. The patients were given supplemental oxygen at the rate of 2 litres/min via nasal prong. They were randomly divided into two groups using sealed envelope techniques: group FICB to receive fascia iliaca compartment block and group FNB to receive femoral nerve block. An experienced anesthesiologist performed all the blocks. Before performing the blocks, the baseline visual analog pain scale (VAS) $(0=$ no pain to $10=$ maximal pain) was clearly explained to the patients and baseline was noted.
The FICB was performed using the approach first described by Dalens et al. ${ }^{5} \mathrm{~A}$ line was drawn on the skin from pubic tubercle to anterior superior iliac spine and divided into three parts. The puncture site was marked, $1-3 \mathrm{~cm}$ distal to the point where the middle and lateral third to this line met. Femoral artery was identified medial to the intended puncture site. Identification of the correct compartment was based on two 'pops' followed by loss of resistance; first 'pop' felt indicated piercing through fascia lata and the second fascia iliaca. We used short beveled $22 \mathrm{G}$ regional analgesia needle. A total of $30 \mathrm{ml}$ of $1.5 \%$ lignocaine with adrenaline was used.

For FNB; the needle was introduced $1 \mathrm{~cm}$ lateral to the femoral artery and $1 \mathrm{~cm}$ below the inguinal ligament. A total of $15 \mathrm{ml}$ 1.5\% lignocaine with adrenaline was injected slowly after a negative aspiration test.

After performing the study blocks the patients were kept in supine position for at least 20 min before shifting them to the operation theatre. On arrival in the theatre, the patients were made to be on sitting position with the help of operation theatre assistants while the skeletal traction was maintained. VAS was enquired and noted and if the patient reported VAS of 4 or more during placement in the sitting position procedure was stopped and inj. fentanyl 1 microgram/kg was given intravenously in the form of rescue analgesia before attempting to reposition again. Once the patient was in sitting position SAB was administered in L 3-4 inter space using $2.4-3 \mathrm{ml}$ of $0.5 \%$ hyperbaric bupivacaine maintaining strict asepsis. The patient was laid down back to supine position. The level of sensory block was assessed using a sterile needle. Motor block was assessed by grading the motor power of the muscles ( 0 to 5 ). After ascertaining adequate block, patient was shifted to fracture table for operation. Time required to perform SAB (as defined as time from insertion of the spinal needle to complete deposit of drug in the subarachnoid space) was noted. The quality of patient positioning was subjectively rated as unsatisfactory, good or optimal depending on the ease of positioning for SAB. Patient acceptance was evaluated after completion of surgery by using a two-point score: $1=$ good, if necessary, I'Il repeat it and $2=$ bad, I will never repeat it again.

Data were collected and entered in a master chart in MS Excel program. Data were analyzed using the Statistical Package for Social Science (version 11.5 for Windows, SPSS Inc. Chicago University, USA). Unpaired t-test was used to compare continuous data while chi-square test (with yates correction) was used to compare categorical data.

To estimate sample size, a pilot study was done involving 10 patients. The study showed mean reduction of VAS of 3.24 and 2.00 in FICB and FNB receiving patients respectively. The standard deviation was 1.2. Keeping an alpha error of 0.005 and power of 0.8 , the estimated sample size was 15 in each arm. 


\section{RESULTS}

Gender, age, American Society of Anesthesiologists Physical Status (ASA PS) distribution of the patients and time gap between injury and surgery were comparable between the groups (Table:1).

Table 1. Demographic variables, ASA PS and time gap between injury and surgery

\begin{tabular}{|llll|}
\hline & \multicolumn{2}{c}{ Group } & p-value \\
\hline & FICB $(\mathbf{n}=\mathbf{1 5})$ & FNB( $\mathbf{n}=15)$ & \\
\hline $\begin{array}{l}\text { Gender } \\
\text { Male/female }\end{array}$ & $10 / 5$ & $7 / 8$ & 0.26 \\
\hline Mean age (yr) \pm SD & $54.4 \pm 18.5$ & $55.0 \pm 21.6$ & 0.94 \\
\hline ASA I/II & $6 / 9$ & $5 / 10$ & 0.70 \\
\hline $\begin{array}{l}\text { Time gap between injury } \\
\text { (days) and surgery }\end{array}$ & $3.40 \pm 1.24$ & $3.07 \pm 1.10$ & 0.44 \\
\hline
\end{tabular}

Baseline VAS scores at the time of block were comparable between the groups. Mean VAS score at positioning for SAB was signifiantly lower in group FICB than in group FNB.

Performance time for SAB in group FICB was significantly shorter than FNB. Performer rated quality of positioning was significantly better in group FICB than group FNB. Patients acceptance was better in group FICB than in group FNB (Table:2). Rescue analgesia was required in two patients, one from each group. No complications attributable to the study blocks were observed during the post operative period.

Table 2. Comparison of VAS scores, performance time, performer rated quality of positioning, and patient acceptance

\begin{tabular}{|lllc|}
\hline & \multicolumn{2}{c}{ Group } & p-value \\
\hline & FICB $(\mathbf{n}=15)$ & FNB(n=15) & \\
\hline VAS at block & $2.9 \pm 0.2$ & $3.0 \pm 0.4$ & 0.33 \\
\hline VAS at positioning for SAB & $1.0 \pm 1.1$ & $2.1 \pm 0.8$ & 0.005 \\
\hline Time to perform SAB (sec) & $109.6 \pm 28.2$ & $134.8 \pm 31.9$ & 0.02 \\
\hline $\begin{array}{l}\text { Quality of patient positioning } \\
\text { Unsatisfactory/ good or optimal }\end{array}$ & $3 / 12$ & $6 / 9$ & 0.008 \\
\hline
\end{tabular}

No adverse systemic toxicity of lignocaine was noted and neither any vascular puncture nor paresthesia was observed. No complications such as hematoma or persistent paresthesia were observed in patients with both the techniques of blocks within following 24 hours after the operation.

\section{DISCUSSION}

The present study has found that both FICB and FNB facilitate positioning for $S A B$ in patients undergoing surgeries for proximal femoral fractures. However, FICB is significantly more effective than FNB. Reduction in VAS by FICB was double than that of FNB in the present study. This finding is not unexpected as hip receives nerve supply occasionally from sciatic and superior gluteal nerves also. These nerve are not affected by the FNB, explaining less reduction in pain in the group. Similar findings have been reported by other investigators demonstrating FICB to provide excellent pain relief for positioning for $S A B{ }^{6-8}$

One important point to be noted in the present study is that the baseline pain VAS in patients of both the arms is around 3 only, which is lower than what most other investigators have reported. ${ }^{6-9}$ One study has reported low baseline VAS as in our study. ${ }^{10}$ Late presentation to the hospital, late scheduling in the operating list and administration of analgesics are likely reasons for this difference. The mean duration of time between the injury and surgery in the present study is more than 3 days unlike within 24 hours in other studies. ${ }^{6-9}$

Better pain relief and, therefore, better positioning with the FICB is well reflected in the time required for performing the $S A B$. The time required for performing $S A B$ with FICB was almost half a minute less than that of FNB in the present study. The performance time of FNB arm of our study is comparable to the time reported by Sia et al. ${ }^{10}$ However, longer SAB performance time of almost seven min has been reported by Yun et al. following FICB. ${ }^{11}$ Clearly it is difficult to compare this observation with ours since the definition of performance time in their study is quite different from ours. Nevertheless, the fact that there is reduction in the SAB performance time with the FICB is clearly not different. Good analgesia and paralysis of some muscles (eg. quadriceps) following FICB are the likely reasons for more comfortable positioning in the group. ${ }^{11}$ This fact has been further supported by the SAB performer rated quality of positioning and acceptance of the technique by the patients.

Inability to take the actual body weight, use of landmark technique instead of ultrasound guidance are some of the important limitations of the present study.

\section{CONCLUSION}

Our findings demonstrate that fascia iliaca compartment block provides better analgesia than femoral nerve block in terms of facilitating optimal positioning for subarachnoid block in patients undergoing proximal femoral fracture fixation procedure.

\section{ACKNOWLEDGEMENTS}

The authors are thankful to all patients who participated in the study and to the research committee of B.P. Koirala institute of health sciences, Dharan, Nepal. 


\section{REFERENCES}

1. Foss NB, Kehlet H. Mortality analysis in hip fracture patients: Implications for design of future outcomes trial. Br J Anaesth 2005; 94(1):24-29.

2. Heikkinen T, Parker M, Jalovaara P. Hip fractures in Finland and Great Britain- a comparison of patient characteristics and outcomes. Int Orthop 2001; 25(6):349-54.

3. Morau D, Lopez S, Biboulet P, Bernard N, Amar J, Capdevila X. Comparison of continuous 3 -in-1 and fascia iliaca compartment blocks for postoperative analgesia: feasibility, catheter migration, distribution of sensory block, and analgesic efficacy. Reg Anesth Pain Med 2003;28 (4): 309-14.

4. Capdevila X, Biboulet P,Bouregba M, Barthelet $Y$, Rubenovitch J, d'Athis F. Comparison of three-in-one and fascia iliaca compartment blocks in adults: clinical and radiographic analysis. Anesth Analg 1998; 86 (5): 1039-44.

5. Dalens B, Vanneuville G, Tanguy A. Comparison of fascia iliaca compartment block with 3-in-1block in children. Anesth Analg 1989; 69 (6): 705-13.
6. Monzon DG, Iserson KV, Vazquez JA. Single fascia iliaca compartment block for post-hip fracture pain relief. J Emerg Med. 2007; 32 (3): 257-62.

7. Beaudoin FL, Nagdev A, Merchant R, Becker B. Ultrasound guided femoral nerve blocks in elderly patients with hip fracture. American journal of emergency medicine 2010; 28, 76-81.

8. Candal-Couto JJ, Mc Vie JL, Haslam N, Innes AR, Rushmer J. Preoperative analgesia for patients with femoral neck fractures using a modified fascia iliaca block technique. Injury. 2005; 36 (4): 505-10.

9. Haines L, Dickman E, Ayvazyan S, Wu S, Rosenblum D, Likourezos A. Ultrasound guided fascia iliaca compartment block for hip fractures in the emergency department. J Emerg Med. 2012; 43(4): 692-7.

10. Sia S, Pelusio F, Barbagli R, Rivituso C. Analgesia before performing a spinal block in the sitting position in patients with femoral shaft fracture: A comparison between femoral nerve block and intravenous fentanyl. Anesth Analg 2004;99:1221-4.

11. Yun MJ, Kim YH, Han MK, Kim JH, Hwang JW, Do SH. Analgesia before a spinal block for femoral neck fracture: fascia iliaca compartment block. Acta Anaesthesiol Scand. 2009;53(10):1282-7. 